This is a postprint version of the following published document:

J. Montalvo, A. Tapetado, D. S. Montero and C. Vázquez. "New Fiber Supervision Technique for Passive Optical Networks Supporting Mobile Services". IEEE. Photonics Technology Letters, 28 (2015) 4, pp. 501 - 504. Available in http://www.dx.doi.org/10.1109/LPT.2015.2500278

(C) 2015. IEEE. Personal use of this material is permitted. Permission from IEEE must be obtained for all other uses, in any current or future media, including reprinting/republishing this material for advertising or promotional purposes, creating new collective works, for resale or redistribution to servers or lists, or reuse of any copyrighted component of this work in other works. 


\title{
New Fiber Supervision Technique for Passive Optical Networks Supporting Mobile Services
}

\author{
J. Montalvo, A. Tapetado, D. S. Montero and C. Vázquez, Senior Member, IEEE
}

Abstract: In this paper, we propose a new fiber supervision technique for Passive Optical Networks (PON). The new approach allows for real-time in-service fiber fault detection and localization at any individual fiber in the PON even after high splitting ratios and optical amplifiers without Optical Distribution Network (ODN) modifications. It consists of real time permanent power monitoring of dedicated supervision wavelengths transmitted between the Central Office and each remote location. This new proposal relies on synchronized clocks between the test equipment and the remote devices, thus being adequate for its use in fixed-mobile convergent PONs. This novel technique has been successfully demonstrated in a commercial in-service GPON system with 1:64 splitting ratio.

Keywords: Mobile communication, Optical fiber communication, Optical fiber measurements, Optical time domain reflectometry.

\section{INTRODUCTION}

$\mathrm{F}$ IBER technologies are currently being massively deployed in access networks worldwide as they are considered to be a future-proof and cost efficient solution to provide ultra-high speed access $(1-10 \mathrm{~Gb} / \mathrm{s})$ for both fixed and $4 \mathrm{G}-5 \mathrm{G}$ mobile connections. Fiber to the Home/Building (FTTH/B) PONs reached close to 150 million connections worldwide in 2014, having been forecasted to get around 250 million connections reached in 2018 [1]. This rapid expansion of PON systems has brought into focus the need for an optical layer management solution with requirements of cost-effective and enhanced physical layer performance monitoring.

A PON consists of an Optical Line Termination (OLT), located at a Central Office (CO) of the network operator, and several Optical Network Units (ONUs), installed at the customer side. The OLT and ONUs communicate with each other through a point to multi-point fiber topology in the optical distribution network (ODN), see Fig. 1a. In Time-DivisionMultiplexing (TDM) PONs, the ODN is built using power splitters. Optical amplification (OA) may also be used in case of Long-Reach PONs.

Optical Time Domain Reflectometry (OTDR) is a wellestablished and well-known technology for the physical layer

This work was supported by the Spanish Ministry of Economía y Competitividad under grant TEC2012-37983-C03-02, Comunidad de Madrid under grant S2013/MIT-2790 and partially from the European Union under grant agreement n. 318137 ("DISCUS").

J. Montalvo is with Telefónica Investigación y Desarrollo S.A.U., 28050 Madrid, Spain.

e-mail:

julio.montalvogarcía@telefonica.com

A. Tapetado, D. S. Montero and C. Vázquez are with the Electronics Technology Department, Carlos III University of Madrid, 28911 Leganés, Madrid.

e-mail:

atapetad@ing.uc3m.es

dsmontero@ing.uc3m.es

cvazquez@ing.uc3m.es
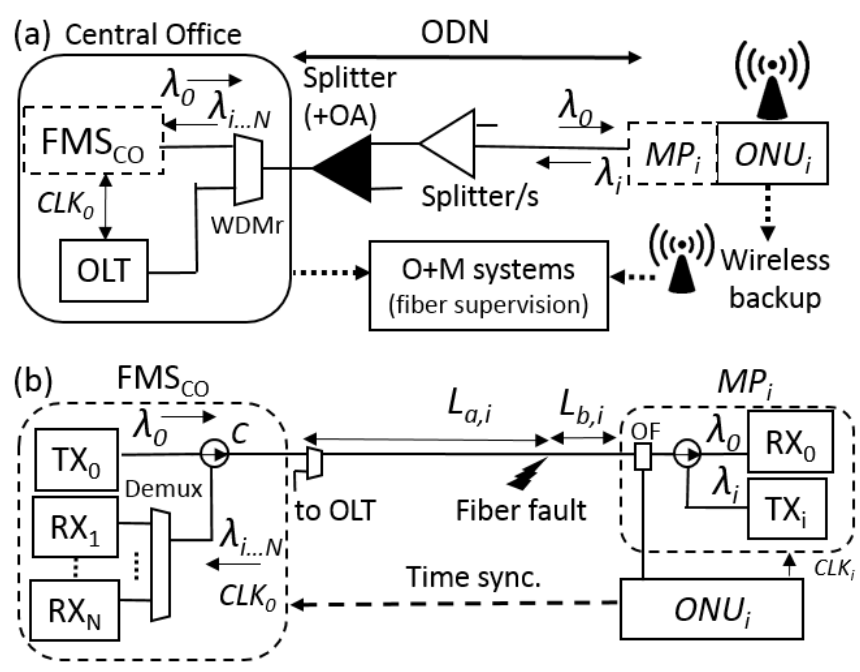

Fig. 1. (a) Generic application scenario of the proposed supervision system for PONs. (b) Detail of the supervision system architecture for $O N U_{i}$. FMS: Fiber Monitoring System. CLK: Clock. C: Circulator. OA: Optical Amplification. $O+M$ : Operations and Maintenance. $M P$ : Monitoring Point.

supervision of optical fibers. Nevertheless, the performance of conventional OTDRs in TDM-PON scenarios is considered insufficient because of the high optical attenuation and the resulting measurement overlapping behind power splitters. A technique has recently been reported for OTDR-based monitoring of TDM-PONs, partially overcoming the limitations of conventional systems [2]; nevertheless, this technique requires some modifications of the ODN, which are highly undesired by network operators in general, as they need to protect the ODN investment in the long-term (decades). Moreover, these ODN modifications may be even unfeasible, as the new components needed may not comply with ODN engineering and installation requirements (size, temperature range, etc...). A pump-probe Brillouin-based OTDR technique has also been reported capable of locating individual fiber faults in TDM-PONs [3]. Anyhow, it does not offer any advantage over conventional OTDRs in case of fiber cuts and it requires high-power optical signals at COs. This may impact the eye safety requirements of network operators. 
In this letter, we propose a new fiber supervision approach overcoming the restrictions of using OTDRs in TDM-PONs. This approach is capable of locating faults up to the end of the fiber connection location behind the power splitters and the amplifier nodes, with no need to modify or to replace the ODN infrastructure.

\section{Description OF THE OptiCAL SUPERVISION SySteM}

\section{A. Architecture}

The proposed architecture consists of a permanent in-line fiber monitoring system using simultaneous real-time bidirectional optical power monitoring at both ends of a fiber path, this is, at the monitoring block $\left(\mathrm{FMS}_{\mathrm{CO}}\right)$ located in the $\mathrm{CO}$ and at the Monitoring Points (MP) installed close to each of the ONUs in the PON, respectively, see Fig. 1a. With the proposed architecture, a logical point to point fiber supervision between the CO and each ONU's MP is achieved in a physical point to multipoint topology.

Fig. $1 \mathrm{~b}$ shows the components employed for the point to point fiber measurement technique. At the $\mathrm{CO}$ side, a monitoring block is required per each PON under monitoring. A wavelength Mux/Demux (WDMr) combines the optical PON data signals of each OLT port with the optical signals of the FMS $_{\mathrm{C} 0}$ monitoring block. A single continuous wave monitoring wavelength is transmitted from the $\mathrm{FMS}_{\mathrm{CO}}$ to all the ONUs using a laser diode (LD) transmitting at $\lambda_{0}\left(\mathrm{TX}_{0}\right)$. At a desired demarcation point close to each $\mathrm{ONU}_{\mathrm{i}}(i=1 \ldots N)$, a Monitoring Point $\left(\mathrm{MP}_{\mathrm{i}}\right)$ is installed that includes an optical filter (OF) which combines both monitoring and data signals. Each $\mathrm{MP}_{\mathrm{i}}$ also comprises a receiver $\left(\mathrm{RX}_{0}\right)$ which is used for measuring the optical power received at $\lambda_{0}$, and a LD transmitting a continuous wave $(\mathrm{CW})$ signal at $\lambda_{i}\left(\mathrm{TX}_{\mathrm{i}}\right)$, being specific for each ONU. Inside the $\mathrm{FMS}_{\mathrm{CO}}$ and each $\mathrm{MP}_{\mathrm{i}}$, both the transmitted and received signals are delivered to the corresponding optical ports by using an optical circulator (C). Time synchronization between a clock located at the $\mathrm{CO}\left(\mathrm{CLK}_{0}\right)$ and a clock located at each $\mathrm{ONU}_{\mathrm{i}}\left(\mathrm{CLK}_{\mathrm{i}}\right)$ is usually required in the PON for mobile base stations for a proper operation of the mobile system. This mechanism is also used in our proposed supervision system approach. The estimated cost increase for a mobile PON connection due to the elements required by the proposed supervision technique is as low as $0.9 \%$, thus the technique is cost-effective in the considered scenarios.

\section{B. Principle of operation}

The proposed architecture uses the principle of operation reported in [4] for point to point measurements in any type of glass and plastic optical fibers. In this paper, we extend the principle of operation for supporting PON topologies based on power splitters and optional optical amplifiers without ODN modifications.

Fig. 1b, depicts a fiber-based optical link between the central monitoring block $\mathrm{FMS}_{\mathrm{CO}}$ and an $\mathrm{ONU}_{\mathrm{i}}$ serving a mobile base station. The following parameters are defined:

- $\lambda_{i}$ : monitoring wavelength, from MPi to CO.

- $\lambda_{0}$ : monitoring wavelength, from CO to all MPs in the
PON.

- $\quad N_{g_{-},}, N_{g_{-} 0}$ : effective refractive index of fiber, at $\lambda_{i}$ and $\lambda_{0}$ respectively.

- $L_{i}=$ total fiber length from $\mathrm{FMS}_{\mathrm{CO}}$ to $\mathrm{MP}_{\mathrm{i}}$.

- $L_{a, i}, L_{b, i}$ : fiber distance from the fiber fault location to $\mathrm{FMS}_{\mathrm{CO}}$ and to $\mathrm{MP}_{\mathrm{i}}$, respectively.

When a fiber fault (fiber bending, cut, disconnection, etc...) causing an attenuation event takes place at a certain time $\mathrm{T}_{0}$ and at a certain distance $L_{a, i}$ from the $\mathrm{CO}$, an optical power attenuation starts propagating from the fiber fault location to both ends of the optical path. This event is detected by the receivers of the monitoring system, $\mathrm{RX}_{\mathrm{i}}$ and $\mathrm{RX}_{0}$, located at the $\mathrm{FMS}_{\mathrm{CO}}$ and the $\mathrm{MP}_{\mathrm{i}}$, respectively, see Fig. $1 \mathrm{~b}$.

An optical power attenuation threshold is configured to generate alarms when the optical power received at both $\mathrm{RX}_{\mathrm{i}}$ and $\mathrm{RX}_{0}$ gets below a specific detected power bound. As the clocks at both ends of the optical path are synchronized, $\mathrm{FMS}_{\mathrm{CO}}$ and $\mathrm{MP}_{\mathrm{i}}$ will generate corresponding alarms assigned to at least one $\mathrm{ONU}_{\mathrm{i}}$ at the following time values:

$$
\begin{aligned}
& T_{a, i}=T_{0}+\frac{L_{a, i}}{c} \cdot N_{g, i} \\
& T_{b, i}=T_{0}+\frac{L_{b, i}}{c} \cdot N_{g, 0}
\end{aligned}
$$

where $c$ is the speed of light in vacuum.

The last term at the second member of both equations is the time-of-flight for a lightwave propagating from the fault location to each fiber end, and their propagation velocities depend on the operating wavelength. $T_{a, i}$ is reached when the power attenuation threshold is surpassed at the $\mathrm{CO}$ in the FMS $_{\mathrm{CO}}$ receiver $\mathrm{RX}_{\mathrm{i}}$, and $T_{b, i}$ is reached when the power attenuation threshold is crossed at $\mathrm{RX}_{0}$ in the remote $\mathrm{ONU}_{\mathrm{i}}$. Both time values can be transmitted to the $\mathrm{O}+\mathrm{M}$ system of the PON operator using a local communication channel at the $\mathrm{CO}$ and a wireless backup communication channel at the ONU.

As both receivers have synchronized clocks available, the absolute value $T_{0}$, which is unknown, can be neglected by subtracting both time values, as follows:

$$
\Delta t_{i}=\left|T_{a . i}-T_{b . i}\right|=\left|\frac{L_{a, i}}{c} \cdot N_{g, i}-\frac{L_{b, i}}{c} \cdot N_{g, 0}\right|
$$

The required synchronization between clocks can be achieved by using protocols such as Precision Time Protocol (PTP) version 2 [5], which can provide a nanosecond-scale time synchronization accuracy, thus allowing fiber fault localizations with a distance resolution below $10 \mathrm{~m}$. The optical length $L_{i}$ between the OLT and each $\mathrm{ONU}_{\mathrm{i}}$ is a known value that can be calculated by the OLT during the ONU ranging process, or measured during ONU installation using a handheld OTDR.

$$
L_{i}=L_{a, i}+L_{b, i}
$$


(a)

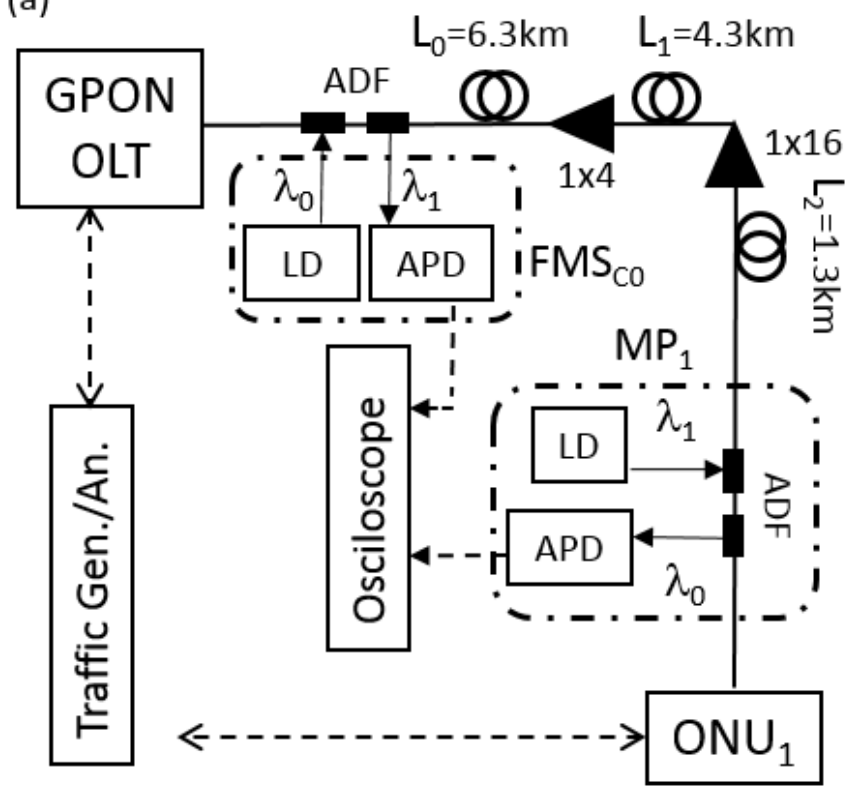

(b)

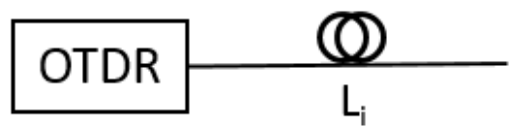

Fig. 2. (a) Experimental set-up. Traffic Generator/Analyzer transmitting two $30 \mathrm{Mb} / \mathrm{s}$ traffic flows to OLT and ONU1. ADF: Add-drop filters. APD: Avalanche Photo-Diode. FMS $\mathrm{C}_{\mathrm{C} 0}$ : Fiber Monitoring System at Central Office. MP1: Monitoring Point 1. (b) Measurement schematic for obtaining the length of fibers $\mathrm{Li}(\mathrm{i}=0,1,2)$ using an OTDR.

From equations (3) and (4), the distance between the $\mathrm{CO}$ and the fiber fault is obtained as follows:

$$
L_{a, i}=\left(c \cdot\left|T_{a, i}-T_{b, i}\right|+L_{i} \cdot N_{g, 0}\right) /\left(N_{g, i}+N_{g, 0}\right)
$$

\section{EXPERIMENTAL SETUP}

\section{A. Test-bed description}

The proposed technique is validated in a real PON scenario. The setup has a commercial Gigabit PON (GPON) OLT, a tabletop ODN with 1:64 splitting ratio using cascaded 1:4 and 1:16 optical power splitters, and an ONU $\left(\mathrm{ONU}_{1}\right)$ with a symmetrical $30 \mathrm{Mb} / \mathrm{s}$ connectivity service configured, see Fig. 2a. In the experiment, available wavelengths in the $\mathrm{C}$ and $\mathrm{L}$ bands, outside the GPON communication wavebands are used.

The ODN fiber lengths are previously obtained by performing individual OTDR point-to-point measurements for each fiber coil $\mathrm{L}_{\mathrm{i}}(\mathrm{i}=0,1,2)$, see Fig. 2b. OTDR measurements are performed using $30 \mathrm{~ns}$ pulses ( $<1 \mathrm{~m}$ resolution) at $1550 \mathrm{~nm}$ during 20 seconds and assuming a fiber effective refractive index $N=1.465$, thus obtaining $\mathrm{L}_{0}=6312 \mathrm{~m}, \mathrm{~L}_{1}=4325 \mathrm{~m}$ and $\mathrm{L}_{2}=1351 \mathrm{~m}$, see Fig. 2a. These measurements are used as a reference in order to analyze the performance of the proposed monitoring technique.

The monitoring blocks at the OLT and ONU sides are built using two in-series Coarse Wavelength Division Multiplexing (CWDM) Add-drop filters (ADF) at nominal wavelengths
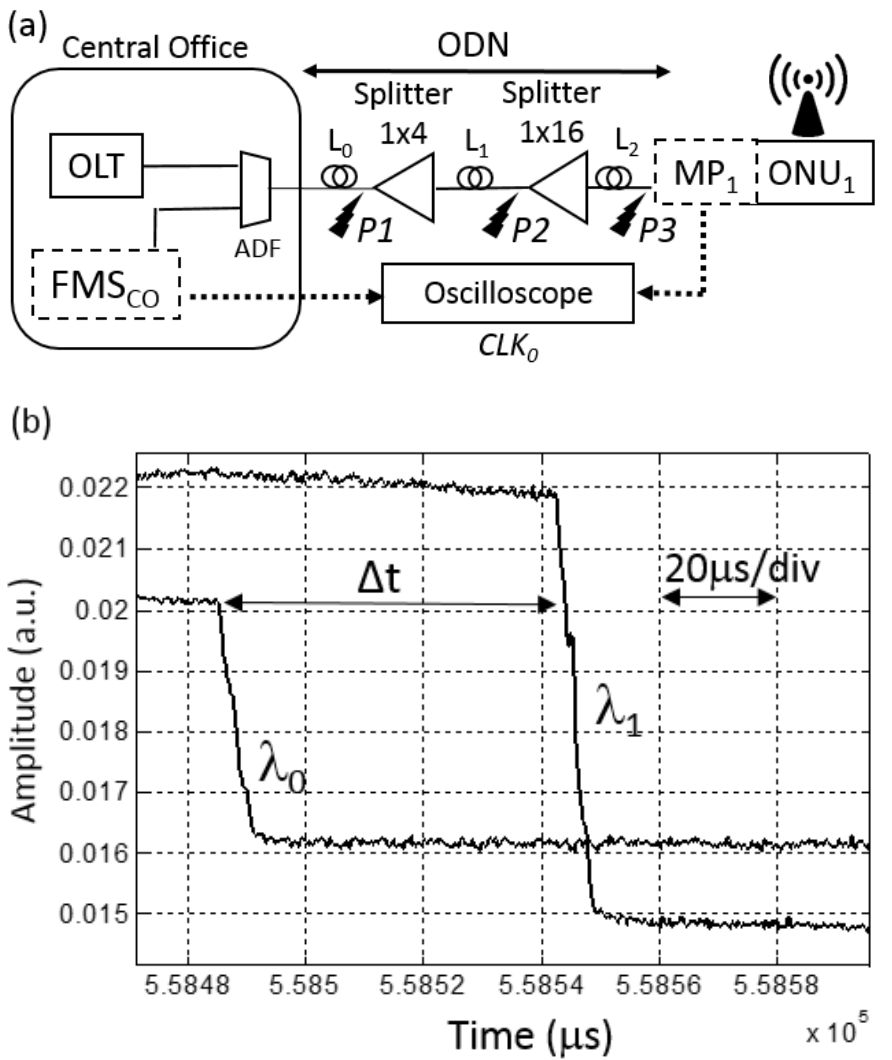

Fig. 3. (a) Schematic of fiber fault emulation at locations P1 (1x4 splitter input), P2 (1x16 splitter input) and P3 (MP 1 input). (b) Measurements during a fiber cut using a fiber cleaver, with fault location at P3. The curve at $\lambda_{0}$ shows the fiber cut detection at $\mathrm{MP}_{1}$ and the curve at $\lambda_{1}$ shows the fiber cut detection at $\mathrm{FMS}_{\mathrm{C} 0}$.

$\lambda_{0}=1550 \mathrm{~nm}$ and $\lambda_{1}=1570 \mathrm{~nm}$, and with corresponding CW LDs transmitting $-0.4 \mathrm{dBm}$ and $-1.1 \mathrm{dBm}$ of optical power, respectively. InGaAs Avalanche Photo-Diodes (APD) with 20 $\mathrm{dB}$ gain factor and $2 \mathrm{MHz}$ bandwidth are used as receivers. The electrical signals at both APDs are measured with an oscilloscope at $4 \mathrm{MSamples} /$ second (MS/s) during 1 second (0.25 $\mathrm{\mu sec} / \mathrm{sample})$, emulating the two synchronized remote processing units of the two monitoring blocks in a single device. A traffic generator-analyzer is employed for bidirectional data transmission between OLT and ONU through the ODN under supervision.

\section{MEASUREMENT RESUlts}

Fiber cuts (using a single mode fiber cleaver) and manual disconnections of SC/APC connectors are carried out and tested at different locations of the ODN in order to evaluate the performance of the proposed monitoring technique in the GPON testbed, see Fig. 3a.

The fiber faults are emulated at the following locations:

- P1: Input of splitter 1x4.

- P2: Input of splitter 1x16.

- P3: Input of Monitoring Point $1\left(\mathrm{MP}_{1}\right)$.

For each fiber fault emulation, the two counterpropagating electrical waves caused by the fault are saved in the oscilloscope memory. A first fiber cut is made at location P3 as 
calibration test to measure the time-of-flight of the light at $\lambda_{1}$ when propagating from $\mathrm{MP}_{1}$ to the APD in the $\mathrm{FMS}_{\mathrm{C} 0}$. The delay time $(\Delta t)$ from Eq. (3) is calculated as the time between the power attenuation starting thresholds at $\lambda_{0}$ and $\lambda_{1}$ measured

TABLE I

MEASUREMENT RESULTS

\begin{tabular}{llll}
\hline \hline Fault location & $\Delta \mathrm{t}(\mu \mathrm{s})$ & $\begin{array}{l}\text { Measured } \\
\text { Distance }(\mathrm{m})\end{array}$ & $\begin{array}{l}\text { Error versus } \\
\text { OTDR }(\mathrm{m})\end{array}$ \\
\hline P1 $^{\mathrm{a}}$ & 2.75 & 6316 & 4 \\
$\mathrm{P} 2$ & 44.50 & 10641 & 4 \\
$\mathrm{P} 3{ }^{\mathrm{a}}$ & 57.25 & 11962 & 26 \\
$\mathrm{P} 3$ & 57.50 & 11988 & $0^{\mathrm{b}}$ \\
\hline \hline
\end{tabular}

${ }^{\mathrm{a}}$ Manual disconnection. ${ }^{\mathrm{b}}$ Calibration.

in the oscilloscope. Fig. 3b shows the measurements at both receivers during a fiber cut made with a fiber cleaver at P3 location. The delay time $(\Delta t)$ is calculated according to Eq. (3) as the time between the power attenuation starting thresholds at $\lambda_{0}$ and $\lambda_{1}$ measured in the oscilloscope.

Measurements with the proposed technique and using an OTDR are shown in Table I. Measurement errors as low as $4 \mathrm{~m}$ for fiber cut localization in a span length of $10 \mathrm{~km}$ are obtained. No impact on the GPON $30 \mathrm{Mb} / \mathrm{s}$ bidirectional traffic between the OLT and the $\mathrm{ONU}_{1}$ is noticed due to the monitoring wavelengths when no fiber fault occurs in the ODN.

\section{SUMMARY AND CONCLUSIONS}

A new fiber fault detection and localization technique for PONs has been successfully demonstrated in a commercial GPON test-bed with no impact in data transmission.

The new technique uses low-cost off-the-shelf optical components and, as opposite to other techniques, avoids the overlapping between centralized measurements such as OTDR traces behind power splitters without any ODN modification. Furthermore, it is compatible with optical amplification in LRPONs.

A measurement accuracy of 10-20 m using photodetectors with a bandwidth of $2 \mathrm{MHz}$ has been demonstrated, detecting and locating individual fiber faults in a PON ODN with 1:64 splitting ratio. This measurement accuracy can be enhanced to $<10 \mathrm{~m}$ by increasing the photodetector bandwidth to $10 \mathrm{MHz}$ and the sampling rate to $20 \mathrm{MS} / \mathrm{s}$.

The new technique relies on a clock synchronization in the remote monitoring points of the $\mathrm{PON}$, thus it is adequate for the individual fiber supervision in ONUs having Mobile Base Stations in the client interface and a wireless backup connection to the $\mathrm{O}+\mathrm{M}$ systems in case of fiber fault.

\section{REFERENCES}

[1] State of FTTx worldwide, IDATE, Dec. 2014.

[2] P.J.Urban et al., "Fiber Plant Manater: An OTDR- and OTM-Based PON Monitoring System”, IEEE Commun. Mag., Vol. 51, n. 2, pp. S9-S15, Feb. 2013.

[3] H. Takahashi et al., "Distributed 32-branched PON Measurement Using Pulsed Pump-Probe Brillouin Analysis", Proc. of $38^{\text {th }}$ ECOC, paper P1.06, Amsterdam, 2012.

[4] D.S. Montero et al., "Fault location in full-duplex plastic optical fiber links using synchronized decay time detection", 22nd International Conference on Plastic Optical Fibers (POF 2013), Sept. 2013.

[5] 1588-2008, IEEE Standard for a Precision Clock Synchronization Protocol for Networked Measurement and Control Systems, IEEE, 07/24/2008. 